\title{
ON CAUCHY PROBLEMS FOR FUZZY DIFFERENTIAL EQUATIONS
}

\section{N. GEORGIOU and I. E. KOUGIAS}

Received 25 February 2002

Existence and uniqueness theorems are proved for Cauchy problems of second-order fuzzy differential equations.

2000 Mathematics Subject Classification: 34A30, 54A40, 26E50, $03 E 72$.

1. Introduction. In 1972, Chang and Zadeh [2] first introduced the concept of fuzzy derivative, followed up ten years later by Dubois and Prade [5], who used the extension principle in their approach. In the mean time, Puri and Ralescu [12] used the notion of $H$-differentiability to extend the differential of set-valued functions to that of fuzzy functions. This led Seikkala [13] to introduce the notion of fuzzy derivative as an extension of the Hukuhara derivative and the fuzzy integral, which was the same as that proposed by Dubois and Prade [3, 4].

Naturally, the investigation of fuzzy differential and integral equations, existence and uniqueness theorems for the solutions of fuzzy initial value problems, drew upon the interest of many researchers of the fuzzy domain. See [1, 6, 7, 8, 9, 10, 11, 12, 13, 14] and the references therein.

In this paper, using the properties of fuzzy integration [8, 9], we examine the existence and uniqueness of initial value problems of second-order fuzzy differential equations:

$$
x^{\prime \prime}(t)=f\left(t, x(t), x^{\prime}(t)\right), \quad x\left(t_{0}\right)=k_{1}, \quad x^{\prime}\left(t_{0}\right)=k_{2} .
$$

2. Preliminaries. A nonempty subset $A$ of $\mathbb{R}^{n}$ is called convex if and only if (1$k) x+k y \in A$ for every $x, y \in A$ and $k \in[0,1]$. By $P_{k}\left(\mathbb{R}^{n}\right)$, we denote the family of all nonempty compact convex subsets of $\mathbb{R}^{n}$.

For $A, B \in P_{k}\left(\mathbb{R}^{n}\right)$, the Hausdorff metric is defined by

$$
d(A, B)=\max \left\{\sup _{a \in A} \inf _{b \in B}\|a-b\| \sup _{b \in B} \inf _{a \in A}\|a-b\|\right\} .
$$

A fuzzy set in $\mathbb{R}^{n}$ is a function with domain $\mathbb{R}^{n}$ and values in $[0,1]$, that is, an element of $[0,1]^{\mathbb{R}^{n}}$ (see $\left.[15,16]\right)$.

A member $u$ of $[0,1]^{\mathbb{R}^{n}}$ is contained in a member $v$ of $[0,1]^{\mathbb{R}^{n}}$ denoted by $u \leq v$ if and only if $u(x) \leq v(x)$, for every $x \in \mathbb{R}^{n}$ (see $[15,16]$ ). 
Let $u, v \in[0,1]^{\mathbb{R}^{n}}$. We define the following fuzzy sets (see $\left.[15,16]\right)$ :

(1) $u \wedge v \in[0,1]^{\mathbb{R}^{n}}$ by $(u \wedge v)(x)=\min \{u(x), v(x)\}$, for every $x \in \mathbb{R}^{n}$ (intersection);

(2) $u \vee v \in[0,1]^{\mathbb{R}^{n}}$ by $(u \vee v)(x)=\max \{u(x), v(x)\}$, for every $x \in \mathbb{R}^{n}$ (union);

(3) $u^{c} \in[0,1]^{\mathbb{R}^{n}}$ by $u^{c}(x)=1-u(x)$, for every $x \in \mathbb{R}^{n}$.

Let $u \in[0,1]^{\mathbb{R}^{n}}$, the a-level set is

$$
\begin{aligned}
& {[u]^{a}=\left\{x \in \mathbb{R}^{n}: u(x) \geq a\right\}, \quad a \in(0,1],} \\
& {[u]^{0}=\operatorname{Cl}\left(\left\{x \in \mathbb{R}^{n}: u(x)>0\right\}\right) .}
\end{aligned}
$$

By $E^{n}$, we denote the family of all fuzzy sets $u \in[0,1]^{\mathbb{R}^{n}}$ (see, e.g., $[12,16]$ ), for which

(i) $u$ is normal, that is, there exists an element $x_{0} \in \mathbb{R}^{n}$ such that $u\left(x_{0}\right)=1$;

(ii) $u$ is fuzzy convex, that is, for any $x, y \in \mathbb{R}^{n}$ and $k \in[0,1]$,

$$
u(k x+(1-k) y) \geq \min \{u(x), u(y)\}
$$

(iii) $u$ is upper-semicontinuous;

(iv) $[u]^{0}$ is compact.

Let $u \in E^{n}$. Then for each $a \in(0,1]$, the $a$-level set $[u]^{a}$ of $u$ is a nonempty compact convex subset of $\mathbb{R}^{n}$, that is, $u \in P_{k}\left(\mathbb{R}^{n}\right)$. Also $[u]^{0} \in P_{k}\left(\mathbb{R}^{n}\right)$.

Let

$$
\begin{gathered}
D: E^{n} \times E^{n} \longrightarrow[0, \infty), \\
D(u, v)=\sup \left\{d\left([u]^{a},[v]^{a}\right): a \in[0,1]\right\},
\end{gathered}
$$

where $d$ is the Hausdorff metric for nonempty compact convex subsets of $\mathbb{R}^{n}$.

It is well known that $\left(E^{n}, D\right)$ is a complete metric space, but it is not locally compact.

Let $u, v \in E^{n}$ and let $c$ be a positive number. The addition $u+v$ and (positive) scalar multiplication $c \cdot u$ in $E^{n}$ are defined in terms of the $a$-level sets by

$$
[u+v]^{a}=[u]^{a}+[v]^{a}, \quad[c \cdot u]^{a}=c[u]^{a},
$$

for every $a \in[0,1]$.

This defines a linear structure on $E^{n}$ such that

$$
D(u+w, v+w)=D(u, v), \quad D(c \cdot u, c \cdot v)=c D(u, v),
$$

for all $u, v \in E^{n}$ and $c>0$.

Let $T=\left[t_{0}, t_{0}+a\right]$ with $a>0$ and $x, y \in E^{n}$.

A mapping $f: T \rightarrow E^{n}$ is differentiable at $t \in T$ if there exists an $f^{\prime}(t) \in E^{n}$ such that the limits

$$
\lim _{h \rightarrow 0^{+}} \frac{f(t+h)-f(t)}{h}, \quad \lim _{h \rightarrow 0^{+}} \frac{f(t)-f(t-h)}{h}
$$

exist and are equal to $f^{\prime}(t)$.

Here the limit is taken in the metric space $\left(E^{n}, D\right)$. At the endpoints of $T$, we consider the one-sided derivatives. 
Let $f: T \rightarrow E^{n}$; the integral of $f$ over $T$, denoted by $\int_{T} f(t) d t$, is defined levelwise by the equation $\left[\int_{T} f(t) d t\right]^{a}=\int_{T} f_{a}(t) d t=\left\{\int_{T} f(t) d t: f: T \rightarrow E^{n}\right.$ is a measurable selection for $\left.f_{a}\right\}$, for all $a \in(0,1]$.

We say that a mapping $f: T \rightarrow E^{n}$ is strongly measurable if, for all $a \in[0,1]$, the set-valued mapping $f_{a}: T \rightarrow P_{k}\left(\mathbb{R}^{n}\right)$ is defined by $f_{a}(t)=[f(t)]^{a}$.

If $f: T \rightarrow E^{n}$ is continuous, then it is integrable (see [8]).

\section{Fuzzy differential equations}

THEOREM 3.1. Let $t_{0} \in[a, b]$ and assume that $f:[a, b] \times E^{n} \times E^{n} \rightarrow E^{n}$ is continuous. Consider the initial value problem (1.1). A mapping $x:[a, b] \rightarrow E^{n}$ is a solution to (1.1) if and only if $x, x^{\prime}$ are continuous and satisfy the integral equation

$$
x(t)=k_{2}\left(t-t_{0}\right)+\int_{t_{0}}^{t}\left(\int_{t_{0}}^{t} f\left(s, x(s), x^{\prime}(s)\right) d s\right) d s+k_{1} .
$$

Proof. Since $f$ is continuous, by [8, Corollary 4.1], it must be integrable. So, for

$$
x^{\prime \prime}(t)=f\left(t, x(t), x^{\prime}(t)\right), \quad t \in[a, b]
$$

we have equivalently

$$
x^{\prime}(t)=\int_{t_{0}}^{t} f\left(s, x(s), x^{\prime}(s)\right) d s+x^{\prime}\left(t_{0}\right)
$$

(see [9, Lemma 3.1]). Since $x^{\prime}\left(t_{0}\right)=k_{2}$, we have

$$
x^{\prime}(t)=\int_{t_{0}}^{t} f\left(s, x(s), x^{\prime}(s)\right) d s+k_{2}
$$

Thus, by [9, Lemma 3.1],

$$
x(t)=\int_{t_{0}}^{t}\left(\int_{t_{0}}^{t} f\left(s, x(s), x^{\prime}(s) d s\right)+k_{2}\right) d s+x\left(t_{0}\right)
$$

equivalently (see [8, Theorem 4.3]),

$$
x(t)=\int_{t_{0}}^{t}\left(\int_{t_{0}}^{t} f\left(s, x(s), x^{\prime}(s)\right) d s\right) d s+\int_{t_{0}}^{t} k_{2} d s+x\left(t_{0}\right) ;
$$

equivalently (see [8, Example 4.1]),

$$
x(t)=\int_{t_{0}}^{t}\left(\int_{t_{0}}^{t} f\left(s, x(s), x^{\prime}(s)\right) d s\right) d s+k_{2}\left(t-t_{0}\right)+x\left(t_{0}\right),
$$


or

$$
x(t)=\int_{t_{0}}^{t}\left(\int_{t_{0}}^{t} f\left(s, x(s), x^{\prime}(s)\right) d s\right) d s+k_{2}\left(t-t_{0}\right)+k_{1} .
$$

EXAMPLE 3.2. The second-order linear nonhomogeneous fuzzy differential equation

$$
x^{\prime \prime}(t)=q_{1} x^{\prime}(t)+q_{2} x(t)+A(t),
$$

where $x, x^{\prime}, A:[a, b] \rightarrow E^{n}$ are continuous functions and $q_{1}, q_{2} \in \mathbb{R} \backslash\{0\}$, with the initial conditions

$$
x\left(t_{0}\right)=k_{1}, \quad x^{\prime}\left(t_{0}\right)=k_{2}, \quad t_{0} \in[a, b],
$$

is equivalent to a Volterra-type fuzzy integral equation

$$
x(t)=\int_{t_{0}}^{t} k(t, s, x(s)) d s+g(t),
$$

where $k:[a, b] \times[a, b] \times E^{n} \rightarrow E^{n}$ and $g(t)=k_{2}\left(t-t_{0}\right)+k_{1}$.

Indeed, by Theorem 3.1, the second-order linear fuzzy differential equation

$$
x^{\prime \prime}(t)=q_{1} x^{\prime}(t)+q_{2} x(t)+A(t), \quad x\left(t_{0}\right)=k_{1}, \quad x^{\prime}\left(t_{0}\right)=k_{2}, \quad t_{0} \in[a, b],
$$

is equivalent to the integral equation

$$
x(t)=\int_{t_{0}}^{t}\left(\int_{t_{0}}^{t} f\left(s, x(s), x^{\prime}(s)\right) d s\right) d s+g(t),
$$

where $f\left(t, x(t), x^{\prime}(t)\right)=q_{1} x^{\prime}(t)+q_{2} x(t)+A(t)$ and $g(t)=k_{2}\left(t-t_{0}\right)+k_{1}$.

Let $k:[a, b] \times[a, b] \times E^{n} \rightarrow E^{n}$ be a map such that $k(t, s, x(s))=\int_{t_{0}}^{t} f\left(s, x(s), x^{\prime}(s)\right) d s$. Then the above integral equation is equivalent to the integral equation (3.11).

Thus, all the theorems on existence and uniqueness proved in $[6,11]$ hold true, equivalently for the fuzzy differential equation examined here.

THEOREM 3.3. Let $f:[a, b] \times E^{n} \times E^{n} \rightarrow E^{n}$ be a continuous map and assume that there exists $k>0$ such that

$$
D\left(f\left(t, x_{1}(t), x_{2}(t)\right), f\left(t, y_{1}(t), y_{2}(t)\right)\right) \leq k D\left(x_{1}(t), y_{1}(t)\right)
$$

for all $t \in[a, b], x_{1}, x_{2}, y_{1}, y_{2}:[a, b] \rightarrow E^{n}$. Then the initial value problem

$$
x^{\prime \prime}(t)=f\left(t, x(t), x^{\prime}(t)\right), \quad x\left(t_{0}\right)=k_{1}, \quad x^{\prime}\left(t_{0}\right)=k_{2}, \quad t_{0} \in[a, b],
$$

has a unique solution on $[a, b]$. 
Proof. Denote by $C\left(J, E^{n}\right)$ the set of all continuous mappings from $J$ to $E^{n}$, where $J$ is an interval in $\mathbb{R}$. We metricize $C\left(J, E^{n}\right)$ by setting

$$
H(x, y)=\sup \{D(x(t), y(t)): t \in J\}
$$

for all $x, y \in C\left(J, E^{n}\right)$. The pair $\left(C\left(J, E^{n}\right), H\right)$ is a complete metric space (see [8]).

Now, let $\left(t_{1}, y_{1}(t), y_{2}(t)\right) \in[a, b] \times E^{n} \times E^{n}$ be arbitrary and let $n>0$ be such that $n^{2} k<1$. We will show that the initial value problem

$$
x^{\prime \prime}(t)=f\left(t, x(t), x^{\prime}(t)\right), \quad x\left(t_{1}\right)=y_{1}, \quad x^{\prime}\left(t_{1}\right)=y_{2}, \quad t_{1} \in[a, b],
$$

has a unique solution on $I=\left[t_{1}, t_{1}+n\right]$.

For $x \in C\left(I, E^{n}\right)$, define $G x$ on $I$ by the equation

$$
G x(t)=y_{2}\left(t-t_{1}\right)+\int_{t_{1}}^{t}\left(\int_{t_{1}}^{t} f\left(s, x(s), x^{\prime}(s)\right) d s\right) d s+y_{1}
$$

Then by [8, Corollary 4.2], $G x \in C\left(I, E^{n}\right)$. Furthermore, by [8, Theorem 4.3] and the Lipschitz condition on $f$, we have

$$
\begin{aligned}
H(G x, G y) & =\sup \{D(G x(t), G y(t)): t \in I\} \\
& =\sup \left\{D \left(\int_{t_{1}}^{t}\left(\int_{t_{1}}^{t} f\left(s, x(s), x^{\prime}(s)\right) d s\right) d s,\right.\right. \\
& \left.\left.\quad \int_{t_{1}}^{t}\left(\int_{t_{1}}^{t} f\left(s, y(s), y^{\prime}(s)\right) d s\right) d s\right): t \in I\right\} \\
& \leq \int_{t_{1}}^{t_{1}+n}\left(\int_{t_{1}}^{t_{1}+n} D\left(f\left(s, x(s), x^{\prime}(s)\right), f\left(s, y(s), y^{\prime}(s)\right)\right) d s\right) d s \\
& \leq \int_{t_{1}}^{t_{1}+n}\left(\int_{t_{1}}^{t_{1}+n} k D(x(s), y(s)) d s\right) d s \\
& \leq k n^{2} H(x, y)
\end{aligned}
$$

for all $x, y \in C\left(I, E^{n}\right)$. Hence, by Banach's contraction mapping theorem, $G$ has a unique fixed point, which by Theorem 3.1 is the desired solution to problem (1.1).

Express $[a, b]$ as a union of a finite family of intervals $I_{k}$ with the length of each interval less than $n$. The preceding paragraph guarantees the existence of a unique solution to problem (1.1) on each interval $I_{k}$. Piecing these solutions together gives us the unique solution on the whole interval $[a, b]$.

EXAMPLE 3.4. The second-order linear nonhomogeneous differential equation

$$
x^{\prime \prime}(t)=q_{1} x(t)+A(t)
$$

where $x, x^{\prime}, A \in C\left([a, b], E^{n}\right)$ and $q_{1} \in \mathbb{R} \backslash\{0\}$, with the initial conditions (3.10), has a unique solution $x \in C\left([a, b], E^{n}\right)$ satisfying the initial conditions. 
Let $f:[a, b] \times E^{n} \times E^{n} \rightarrow E^{n}$ be such that $f\left(t, x(t), x^{\prime}(t)\right)=q_{1} x(t)+A(t), t \in T$. Clearly, the map $f$ is continuous and

$$
\begin{aligned}
D(f & \left.\left(t, x_{1}(t), x_{2}(t)\right), f\left(t, y_{1}(t), y_{2}(t)\right)\right) \\
& =D\left(q_{1} x_{1}(t)+A(t), q_{1} y_{1}(t)+A(t)\right) \\
& =D\left(q_{1} x_{1}(t), q_{1} y_{1}(t)\right) \leq\left|q_{1}\right| D\left(x_{1}(t), y_{1}(t)\right)
\end{aligned}
$$

for all $t \in[a, b]$.

Thus, by Theorem 3.3, the above problem has a unique solution.

THEOREM 3.5. Let $f: T \times E^{n} \times E^{n} \rightarrow E^{n}$ be a continuous map and assume that there exists $k>0$ such that

$$
d\left(\left[f\left(t, x(t), x^{\prime}(t)\right)\right]^{a},\left[f\left(t, y(t), y^{\prime}(t)\right)\right]^{a}\right) \leq k d\left([x(t)]^{a},[y(t)]^{a}\right)
$$

for all $a \in[0,1]$. Then the initial value problem

$$
x^{\prime \prime}(t)=f\left(t, x(t), x^{\prime}(t)\right), \quad x\left(t_{0}\right)=k_{1}, \quad x^{\prime}\left(t_{0}\right)=k_{2}, \quad t_{0} \in T,
$$

has a unique solution.

Proof. Indeed, we have

$$
\begin{aligned}
D(f( & \left.\left.t, x(t), x^{\prime}(t)\right), f\left(t, y(t), y^{\prime}(t)\right)\right) \\
& =\sup \left\{d\left(\left[f\left(t, x(t), x^{\prime}(t)\right)\right]^{a},\left[f\left(t, y(t), y^{\prime}(t)\right)\right]^{a}\right): a \in[0,1]\right\} \\
& \leq k \sup \left\{d\left([x(t)]^{a},[y(t)]^{a}\right): a \in[0,1]\right\} \\
& =k D(x(t), y(t))
\end{aligned}
$$

for all $t \in T$ and $x, y \in E^{n}$.

Thus, by Theorem 3.3, the above problem has a unique solution.

EXAMPLE 3.6. Let $x, A: T \rightarrow E^{n}$ be continuous maps and $q>0$. Then the initial value problem

$$
x^{\prime \prime}(t)=q x(t)+A(t), \quad x\left(t_{0}\right)=k_{1}, \quad x^{\prime}\left(t_{0}\right)=k_{2}, \quad t_{0} \in T,
$$

has a unique solution.

Let $f: T \times E^{n} \times E^{n} \rightarrow E^{n}$ be such that $f\left(t, x(t), x^{\prime}(t)\right)=q x(t)+A(t), t \in T$, and $x(t), x^{\prime}(t) \in E^{n}$. Clearly, the map $f$ is continuous and

$$
\begin{aligned}
d([ & \left.\left.f\left(t, x(t), x^{\prime}(t)\right)\right]^{a},\left[f\left(t, y(t), y^{\prime}(t)\right)\right]^{a}\right) \\
& \leq d\left([q x(t)+A(t)]^{a},[q y(t)+A(t)]^{a}\right) \\
& =d\left([q x(t)]^{a}+[A(t)]^{a},[q y(t)]^{a}+[A(t)]^{a}\right) \\
& =d\left([q x(t)]^{a},[q y(t)]^{a}\right) \\
& \leq q d\left([x(t)]^{a},[y(t)]^{a}\right)
\end{aligned}
$$

for all $t \in T, x, y \in E^{n}$, and $a \in[0,1]$. 
Thus the above problem has a unique solution.

\section{REFERENCES}

[1] J. J. Buckley and T. Feuring, Fuzzy differential equations, Fuzzy Sets and Systems 110 (2000), no. 1, 43-54.

[2] S. S. L. Chang and L. A. Zadeh, On fuzzy mapping and control, IEEE Trans. Systems Man Cybernet. 2 (1972), 30-34.

[3] D. Dubois and H. Prade, Towards fuzzy differential calculus. I. Integration of fuzzy mappings, Fuzzy Sets and Systems 8 (1982), no. 1, 1-17.

[4] _ Towards fuzzy differential calculus. II. Integration on fuzzy intervals, Fuzzy Sets and Systems 8 (1982), no. 2, 105-116.

[5] _ _ Towards fuzzy differential calculus. III. Differentiation, Fuzzy Sets and Systems 8 (1982), no. 3, 225-233.

[6] D. N. Georgiou and I. E. Kougias, On fuzzy Fredholm and Volterra integral equations, J. Fuzzy Math. 9 (2001), no. 4, 943-951.

[7] _ Bounded solutions for fuzzy integral equations, Int. J. Math. Math. Sci. 31 (2002), no. 2, 109-114.

[8] O. Kaleva, Fuzzy differential equations, Fuzzy Sets and Systems 24 (1987), no. 3, 301-317.

[9] _ The Cauchy problem for fuzzy differential equations, Fuzzy Sets and Systems 35 (1990), no. 3, 389-396.

[10] J. Y. Park and H. K. Han, fuzzy differential equations, Fuzzy Sets and Systems 110 (2000), 69-77.

[11] J. Y. Park and J. U. Jeong, A note on fuzzy integral equations, Fuzzy Sets and Systems 108 (1999), no. 2, 193-200.

[12] M. L. Puri and D. A. Ralescu, Differentials for fuzzy functions, J. Math. Anal. Appl. 64 (1978), 369-380.

[13] S. Seikkala, On the fuzzy initial value problem, Fuzzy Sets and Systems 24 (1987), no. 3, 319-330.

[14] S. Song and C. Wu, Existence and uniqueness of solutions to Cauchy problem of fuzzy differential equations, Fuzzy sets and Systems 110 (2000), 55-67.

[15] L. A. Zadeh, Fuzzy sets, Information and Control 8 (1965), 338-353.

[16] H. J. Zimmermann, Fuzzy Set Theory and Its Applications, 2nd ed., Kluwer Academic publishers, Massachusetts, 1991.

D. N. Georgiou: Department of Mathematics, Faculty of Sciences, University of Patras, 26500 Patras, Greece

E-mail address: georgiou@math. upatras.gr

I. E. Kougias: Department of Accounting, School of Business Administration and Economics, Technological Educational Institute of Epirus, 48100 Preveza, Greece

E-mail address: kougias@teiep.gr 


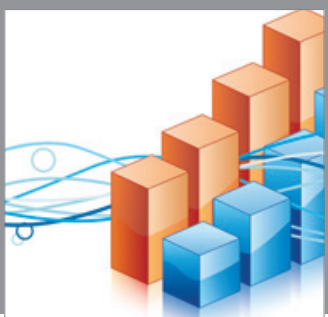

Advances in

Operations Research

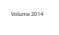

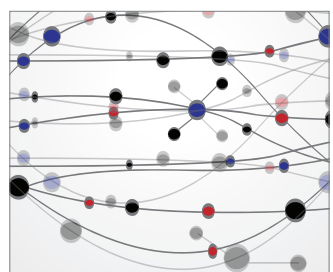

\section{The Scientific} World Journal
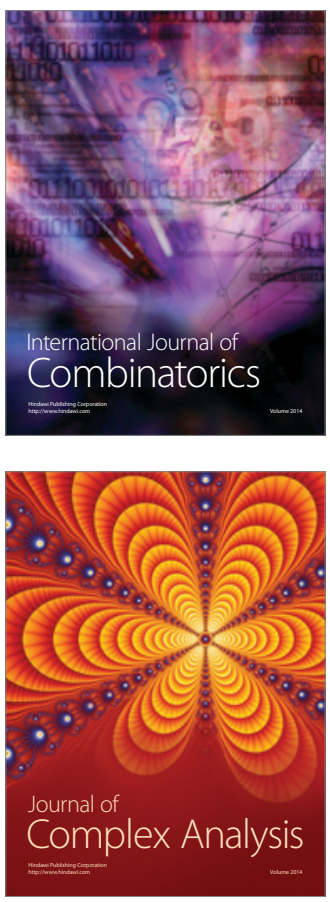

International Journal of

Mathematics and

Mathematical

Sciences
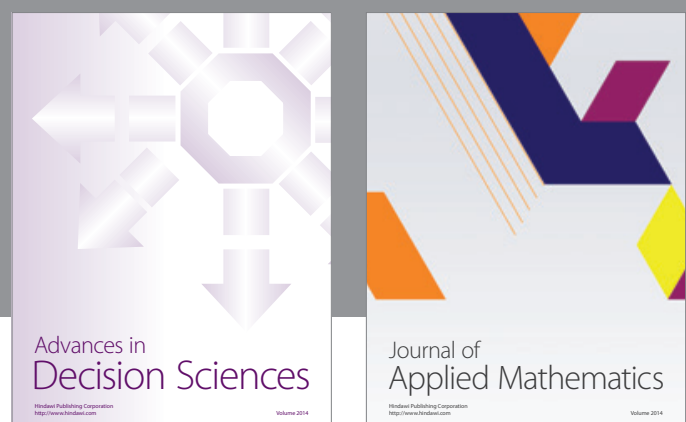

Journal of

Applied Mathematics
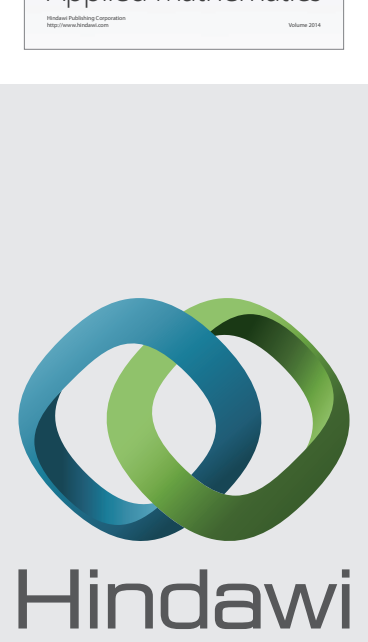

Submit your manuscripts at http://www.hindawi.com
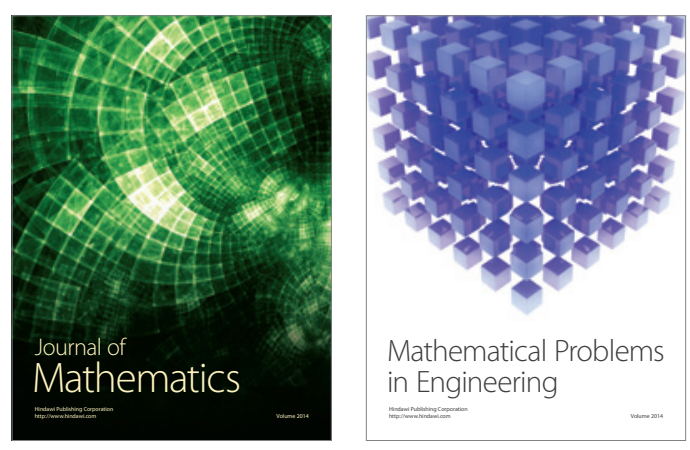

Mathematical Problems in Engineering
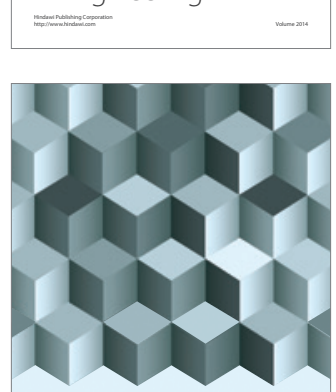

Journal of

Function Spaces
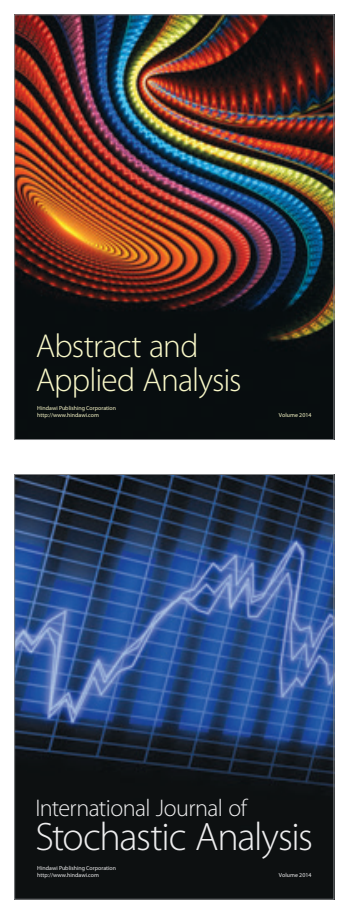

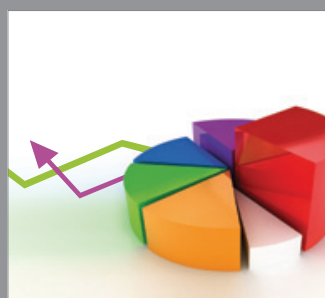

ournal of

Probability and Statistics

Promensencen
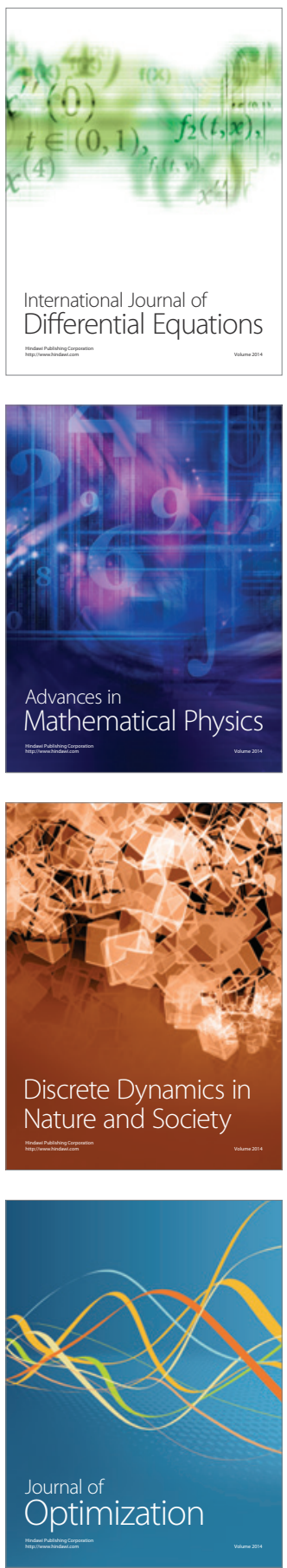